\title{
Defining the popliteal fossa by bony landmarks and mapping of the courses of the neurovascular structures for application in popliteal fossa surgery
}

\author{
Kelsi Greenwood ${ }^{1}$, Reinette van Zyl ${ }^{1}$, Natalie Keough ${ }^{1,2}$, Erik Hohmann ${ }^{1,3,4}$ \\ ${ }^{1}$ Department of Anatomy, School of Medicine, Faculty of Health Sciences, University of Pretoria, Pretoria, South Africa, ${ }^{2}$ Department of Anatomy and \\ Cellular Biology, College of Medicine and Health Sciences, Khalifa University, Abu Dhabi, ${ }^{3}$ Department of Orthopaedic Surgery and Sports Medicine, \\ Burjeel Hospital for Advanced Surgery, Dubai, United Arab Emirates, ${ }^{4}$ Medical School, University of Pretoria, Pretoria, South Africa
}

\begin{abstract}
Surgical access to the posterior knee poses a high-risk for neurovascular damage. The study aimed to define the popliteal fossa by reliable bony landmarks and comprehensively mapping the neurovascular structures for application in posterior knee surgery. Forty-five (20 male, 25 female) embalmed adult cadaveric knees were included. The position of the small saphenous vein (SSV), medial cutaneous sural nerve (MCSN) and lateral cutaneous sural nerv (LCSN), tibial nerve (TN) and common fibular nerve (CFN) nerves, and popliteal vein (PV) and popliteal artery (PA) were determined in relation to either medial (MFE) or lateral (LFE) femoral epicondyles, medial (MTC) and lateral (LTC) tibial condyles and the midpoint between the MFE and MTC and LFEF and LTC. The distance between the MFE and the PA, PV, TN, MCSN, and SSV was $38.4 \pm 12.1 \mathrm{~mm}, 38.4 \pm 12.9 \mathrm{~mm}, 39.4 \pm 10.2 \mathrm{~mm}, 39.2 \pm 14.0 \mathrm{~mm}$ and $37.6 \pm 12.5 \mathrm{~mm}$ respectively for males and $34.6 \pm 4.9 \mathrm{~mm}$, $32.8 \pm 5.6 \mathrm{~mm}$ and $38.0 \pm 8.1 \mathrm{~mm} 38.8 \pm 10.1 \mathrm{~mm}$ and $37.9 \pm 8.2 \mathrm{~mm}$ respectively for females. The distance between LFE and the CFN and LCSN was $13.4 \pm 8.2 \mathrm{~mm}$ and $24.9 \pm 7.3 \mathrm{~mm}$ respectively for males and $8.4 \pm 9.1 \mathrm{~mm}$ and $18.4 \pm 10.4 \mathrm{~mm}$ respectively in females. This study defined the popliteal fossa by reliable bony landmarks and provided a comprehensive map of the neurovascular structures and will help to avoid injuries to the important neurovascular structures.
\end{abstract}

Key words: Knee, Tibial nerve, Popliteal artery, Anatomy

Received July 13, 2020; Revised September 1, 2020; Accepted September 10, 2020

\section{Introduction}

The posterior knee holds a high risk for surgical access due to the dense field of neurovascular structures located in and around the popliteal fossa [1]. Because of the inherent

\footnotetext{
Corresponding author:

Erik Hohmann (10)

School of Medicine, Faculty of Health Sciences, University of Pretoria,

Pretoria 0001, South Africa

E-mail: ehohmann@hotmail.com
}

high risk and unfamiliar relevant surgical anatomy, many surgeons avoid this region altogether $[1,2]$. The main limitation to a direct open posterior approach to the knee is the risk posed to the neurovascular structures [1]. An in-depth knowledge of the surgical anatomy of these neurovascular structures will assist in minimizing the risk of neurovascular damage.

Indications for direct posterior surgical access to the knee include posterior tibial plateau fractures, tibial bony avulsions with associated posterior cruciate ligament injury and repair of the popliteal neurovascular structures [2]. There are several potential pathological conditions, such as cysts or 
mass formation, including neoplasms and abscesses that require surgical treatment which may affect the neurovascular structures in the popliteal fossa [3]. To address these conditions, a posterior surgical approach to the knee is required. This approach is challenging and requires detailed knowledge of the neurovascular structures, as well as the possible variations of the posterior knee [1].

For surgical approaches to the posterior knee, it would be beneficial if surgeons could use reliable anatomic landmarks, such as the femoral epicondyle, to measure the distances between the structures that are potentially endangered by surgery, in particular the popliteal artery and vein and the tibial and common fibular nerves. This would reduce the risk of permanent damage, potentially make surgical planning easier and allow safe and effective surgery. Hence, the purpose of this study was to define the popliteal fossa by reliable bony landmarks and provide a comprehensive map of the neurovascular structures for posterior popliteal fossa knee surgery.

\section{Material and Methods}

This study was designed as a cross-sectional, quantitative study. Formalin preserved cadaveric specimens were obtained from the Department of Anatomy, University of Pretoria, Pretoria, South Africa (Ethics number: 82/2019) (blinded for review). These specimens were donated and used for the anatomy courses for medical and dental students. Prior to these courses, specimens were dissected by one independent researcher. These embalmed cadavers are used for training and research and comply with all the requirements set out in the National Health Act 63 of 2003. Knees were only included if there was no obvious macroscopic damage, no observed previous trauma with signs of corrective surgery, and no visible pathology or fractures of the knee joint. Knees were excluded if evidence existed of previous surgery to the popliteal fossa including vascular repair or bypass surgery, vein stripping or nerve repair. Specimens with Baker's cysts and severe degenerative osteoarthritis with osteophyte formation and any macroscopic neurovascular were also excluded. The sample of the study consisted of 45 adult embalmed cadaveric knees. The mean age of the cadavers was $65.8 \pm 17.4$ years. Of the sample of 45 adult knees, 20 were male and 25 were female. There were 28 left knees used and 17 right knees.

\section{Dissection technique and measurements}

A vertical incision was made in the skin of the posterior knee extending from the mid-thigh to a point just distal to the knee joint. The skin was reflected laterally to expose the defined borders of the popliteal fossa. Soft tissue, including fascia and subcutaneous fat was removed around the distal femur and proximal tibia both medially and laterally. Four bony landmarks were palpated and a pin was placed at each of these landmarks, namely: the medial most point of the medial epicondyle of the femur (MFE), the medial most point of the medial condyle of the tibia (MTC), the lateral most point of the lateral epicondyle of the femur (LEF) and the lateral most point of the lateral condyle of the tibia (LTC). A pin was used to mark all four bony landmarks (Fig. 1). The neurovascular structures (the small saphenous vein [SSV], medial cutaneous sural nerve [MCSN], lateral cutaneous sural nerve [LCSN], tibial nerve [TN], common fibular nerve [CFN],

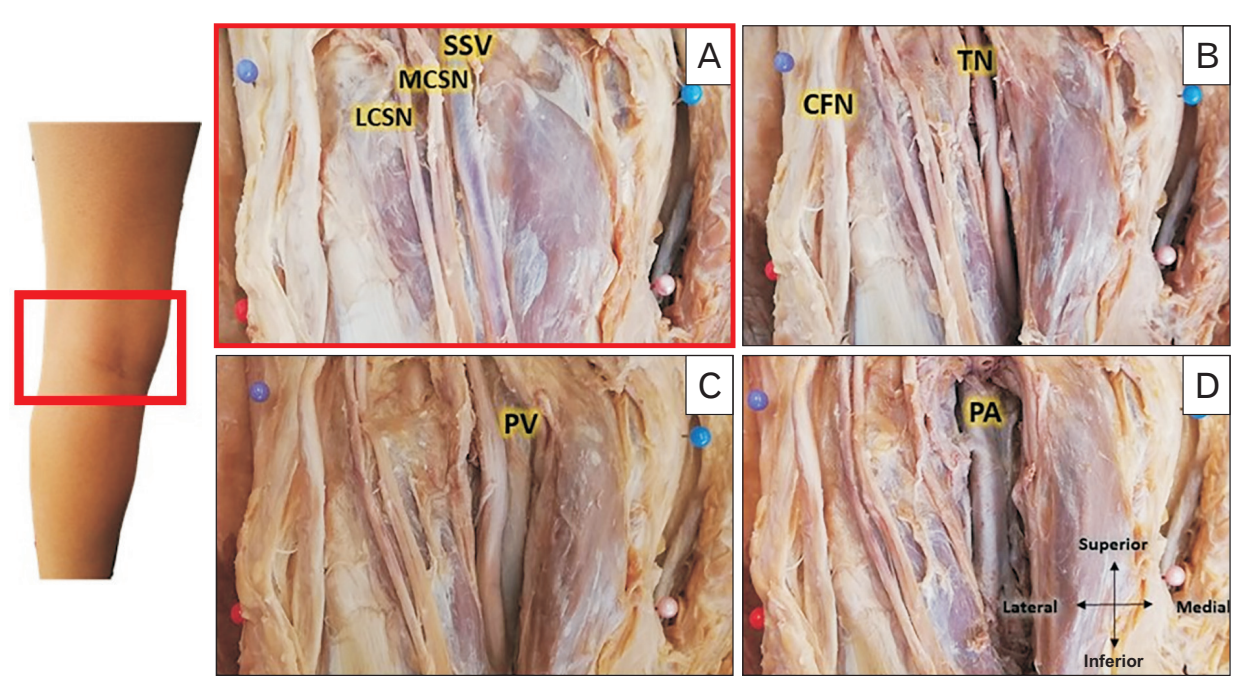

Fig. 1. The dissected left popliteal fossa. The superficial to deep dissection of the posterior cadaveric knee to expose the neurovascular structures. (A) The exposure of the LCSN, MCSN and the SSV. (B) The exposure of the CFN and TN. (C) The dissection to expose the PV. (D) The dissected PA. CFN, common fibular nerve; LCSN, lateral cutaneous sural nerve; MCSN, medial cutaneous sural nerve; PA, popliteal artery; PV, popliteal vein; SSV, small saphenous vein; TN, tibial nerve. 
popliteal vein $[\mathrm{PV}]$ and popliteal artery $[\mathrm{PA}]$ ) were exposed from superficial to deep, with a photograph taken at each level to capture the relevant structures in situ. Great care was taken to ensure that both distance and angles of each photograph were identical by using a tripod. Each photograph included a scale and the photographs were taken perpendicular to the longitudinal axis of the femur and parallel to the floor (Fig. 1). The following distances between the four bony landmarks were then measured with a sliding calliper, calibrated to 0.01 millimetres (Fig. 2):

- M1 distance between the MFE and LFE

- M2 distance between the MTC and LTC

- M3: distance between the MFE and MTC

- M4: distance between the LFE and LTC

The rectangle created by connecting the four bony landmarks was defined as the popliteal fossa (Fig. 2). The photographs were imported into ImageJ software (National Institute of Health, Bethesda, MD, USA) and digital measurements of the distance between the predefined neurovascular structures and the pinned bony landmarks were taken (Table 1). The scale was calibrated using ImageJ on each individual image. All neurovascular structures were measured from the medial bony landmarks to their medial borders, except for the CFN and LCSN, which were measured from the lateral bony landmarks to their lateral borders (Figs. 3-5). The point of measurement of the bony landmark was taken from the centre of the pinhead.
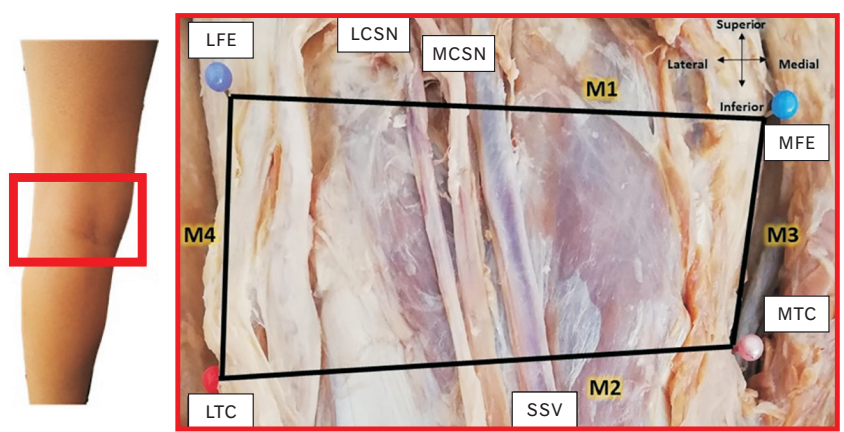

Fig. 2. The definition of the left popliteal fossa. The LFE and the MFE were identified and a pin was placed. Similarly, the LTC and the MTC were identified and marked with a pin. The four pinned bony landmark points were connected to demarcate the area of the popliteal fossa (M1-4). LCSN, ateral cutaneous sural nerve; LFE, lateral most point of the lateral epicondyle of the femur; LTC, lateral most point of the lateral condyle of the tibia; MCSN, medial cutaneous sural nerve; MFE, medial most point of the medial epicondyle of the femur; MTC; medial most point of the medial condyle of the tibia; SSV, small saphenous vein.
Table 1. Measurement descriptions

\begin{tabular}{|ll}
\hline Measurement & \multicolumn{1}{c}{ Description } \\
\hline M5 & Distance between MFE and medial border of the SSV \\
\hline M6 & Distance between MP and medial border of the SSV \\
\hline M7 & Distance between MTC and the medial border of the SSV \\
\hline M9 & Distance between MFE and the medial border of MCSN \\
\hline M10 & Distance between MP and the medial border of MCSN \\
\hline M11 & Distance between MTC and the medial border of MCSN \\
\hline M12 & Distance between LFE and the lateral border of the LCSN \\
\hline M13 & Distance between LTC and the lateral border of the LCSN \\
\hline M14 & Distance between MFE and the medial border of TN \\
\hline M15 & Distance between MP and the medial border of TN \\
\hline M16 & Distance between MTC and the medial border of TN \\
\hline M17 & Distance between LFE and the lateral border of CFN \\
\hline M18 & Distance between MP and the lateral border of CFN \\
M19 & Distance between LTC and the lateral border of CFN \\
M20 & Distance between MFE and the medial border of PV \\
\hline M21 & Distance between MP and the medial border of the PV \\
\hline M22 & Distance between MTC and the medial border of PV \\
\hline M23 & Distance between MFE and the medial border of the PA \\
M24 & Distance between MP and the medial border of the PA \\
M25 & Distance between MTC and the medial border of the PA \\
\hline
\end{tabular}

All measurements taken in mm. MFE, medial most point of the medial epicondyle of the femur; SSV, small saphenous vein; MP, midpoint; MTC, medial most point of the medial condyle of the tibia; MFE, medial most point of the medial epicondyle of the femur; MCSN, medial cutaneous sural nerve; LFE, lateral most point of the lateral epicondyle of the femur; LCSN, lateral cutaneous sural nerve; LTC, lateral most point of the lateral condyle of the tibia; TN, tibial nerve; CFN, common fibular nerve; PV, popliteal vein; PA, popliteal artery.

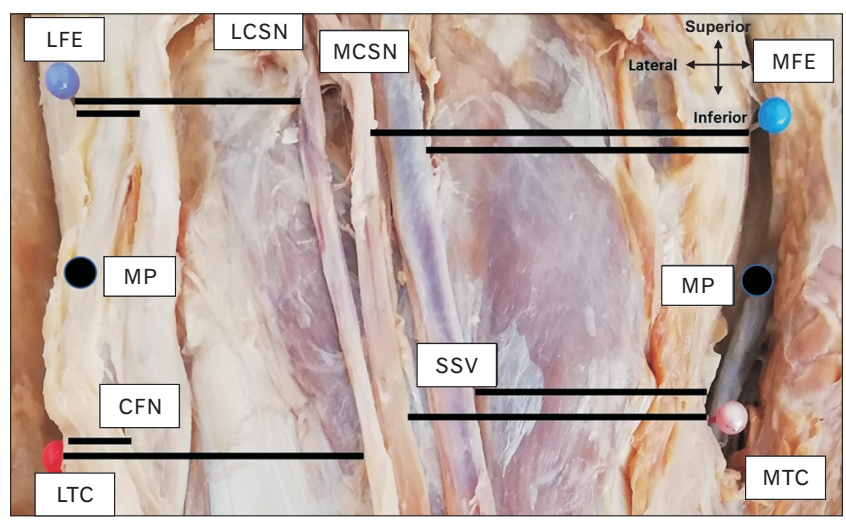

Fig. 3. The measurements of the MCSN and SSV were performed from the MFE, MTC and the MP between these landmarks. The measures for the LCSN and CFN were performed from the LFE, LTC and MP between these landmarks. Measures for LFE, LTC, MFE and MTC are shown. CFN, common fibular nerve; LCSN, lateral cutaneous sural nerve; LFE, lateral most point of the lateral epicondyle of the femur; LTC, lateral most point of the lateral condyle of the tibia; MCSN, medial cutaneous sural nerve; MFE, medial most point of the medial epicondyle of the femur; MP, midpoint; MTC; medial most point of the medial condyle of the tibia; SSV, small saphenous vein. 


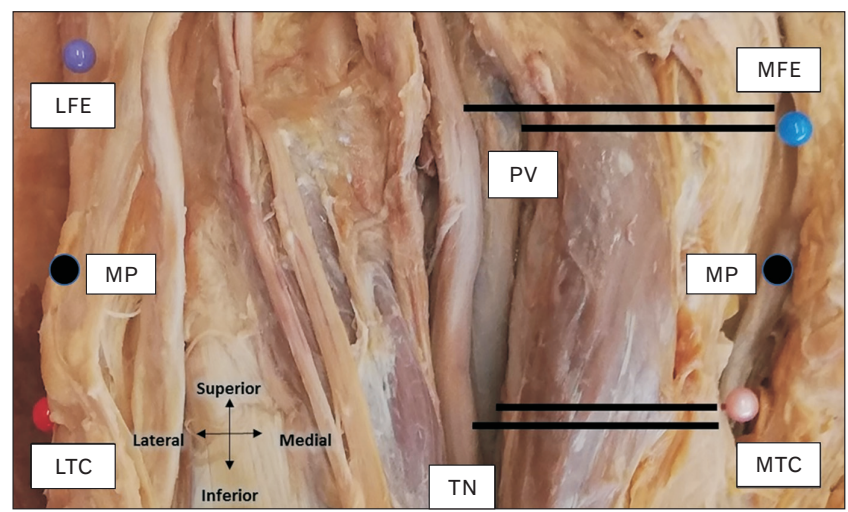

Fig. 4. The PV and TN were measured from the MFE, MTC and the MP between these landmarks. LFE, lateral most point of the lateral epicondyle of the femur; LTC, lateral most point of the lateral condyle of the tibia; MFE, medial most point of the medial epicondyle of the femur; MP, midpoint; MTC; medial most point of the medial condyle of the tibia; PV, popliteal vein; TN, tibial nerve.

\section{Statistical analysis}

Descriptive statistics were used for the distance measurements. Mean length, standard deviation, 95\% confidence intervals and minimum and maximum values were calculated. Normal data distribution was assessed with the ShapiroWilks Test. Homogeneity of variance was verified with Levene's test. A series of unpaired t-tests was used to compare sex and laterality differences. If significant differences were observed between male and female distance measures were analysed separately. Intra- and inter-rater reliability (intraclass correlation coefficients, ICC) were established by repeating the measures on two consecutive days in five cadaveric specimens. The algorithm of Landis and Koch [4] was used to assess the rate of agreement. Values above 0.80 represented excellent agreement, values between $0.62-0.79$ were considered good agreement, values between $0.41-0.61$ indicated moderate agreement, and values below 0.4 suggested fair to poor agreement [4]. Pearson correlations coefficients were used to determine the relationships between the bony landmarks and the distance from the neurovascular structures. Pearson correlations coefficients were also used determined to the relationships between weight/height and the anatomic measures. For this part of the study an a-priori sample size calculation was performed. Using $G^{\star}$ Power 3.1.9.2 (G*Power, Heinrich Heine University, Duesseldorf, Germany) the following variables were used: $\mathrm{H} 1$ corr $p \_a b$ of $0.1, \mathrm{H} 1$ corr $p \_$ac of -0.46 , alpha of 0.05 , beta of 0.8 and a critical $z$ of -1.644 the minimum sample size was calculated to be $n=41$. Based All analyses were conducted using STATA SE (Version

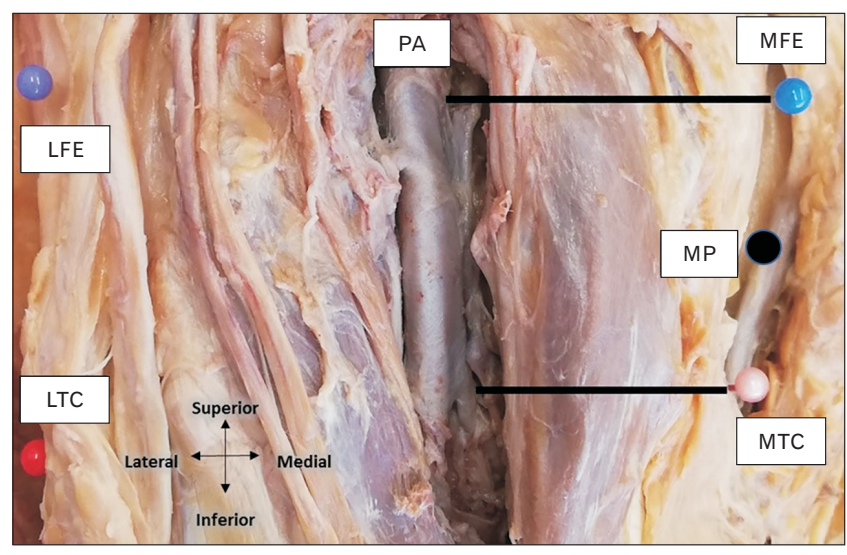

Fig. 5. The PA was measured from the MFE, MTC and the MP between these landmarks. LFE, lateral most point of the lateral epicondyle of the femur; LTC, lateral most point of the lateral condyle of the tibia; MFE, medial most point of the medial epicondyle of the femur; MP, midpoint; MTC; medial most point of the medial condyle of the tibia; PA, popliteal artery.

12.0; StataCorp, College Station, TX, USA) for Windows.

\section{Results}

Intra- and inter-rater reliability (ICC) were scored between three raters and ranged from 0.89-0.98 for interrater reliability and 0.95-0.97 for intra-rater reliability, which represents excellent agreement values [4]. All data were normally distributed and unpaired t-tests did not demonstrate statistical significance between left and right knees $(P=0.36)$. There were significant differences between male and female knees $(P=0.01)$ and therefore, the statistical analysis was reported separately for males and female.

The distances between MFE and LFE, MTC and LTC, MFE and MTC, and LFE and LTC are summarized in Table 2. All distances were observed to be longer in males compared to females with the distance between MFE and LFE on average $8 \%$ longer, between MTC and LTC and between MFE and MTC 7\% longer, and between MFE and LTC only 3\% longer.

Measurements were taken from the medial bony landmarks to the medial lying structures namely the SSV, MCSN, TN, PV, and PA (Table 3). It is important to note that the values for M5-M10 and M14-M16 for the SSV, MCSN and TN are very similar between male and female. This shows a difference in proportion between male and female neurovascular layout for these three structures. The male bony landmark values were consistently larger than the female (Table 2), 
meaning that in males the SSV, MCSN and TN will lie more medially when compared as a proportion. For example, M5 for both male and female was very similar, around $37 \mathrm{~mm}$, however, the male femoral epicondylar distance (M1) was $84.8 \mathrm{~mm}$ and the female $78.9 \mathrm{~mm}$. The male epicondylar distance being significantly larger than the female means that the SSV will lie more medially in the male popliteal fossa.

The LCSN and CFN were measured from the lateral bony landmarks. The CFN in male and female has a mean minimum distance from the lateral landmarks of $0.0 \mathrm{~mm}$ (Table 4). This shows that the CFN ran over those landmarks in a few specimens. Of the sample of 45 knees, there was a $22.2 \%(n=10 / 45)$ occurrence of the CFNs coursing over at least one of the lateral bony landmarks. Neither the CFN nor LCSN displayed proportional differences between male and female as noted with the SSV, MCSN, and TN.

The medial or lateral deviations where calculated for each neurovascular structure. The deviation is between the femo-

Table 2. The definition of the popliteal fossa: the distances between the four bony landmarks

\begin{tabular}{|c|c|c|c|c|c|c|c|c|c|c|}
\hline \multirow{2}{*}{ Measurement } & \multicolumn{5}{|c|}{ Male } & \multicolumn{5}{|c|}{ Female } \\
\hline & $\mathrm{N}$ & Min & Max & Mean \pm SD & $95 \% \mathrm{CI}$ & $\mathrm{N}$ & Min & Max & Mean \pm SD & $95 \% \mathrm{CI}$ \\
\hline MFE-LFE & 20 & 77.5 & 98.3 & $84.8 \pm 5.4$ & 2.4 & 25 & 62.2 & 94.3 & $78.9 \pm 6.4$ & 2.5 \\
\hline MTC-LTC & 20 & 70.2 & 97.1 & $80.3 \pm 6.6$ & 2.9 & 25 & 59.1 & 91.5 & $75.4 \pm 7.9$ & 3.1 \\
\hline MFE-MTC & 20 & 31.6 & 54.0 & $40.5 \pm 6.3$ & 2.8 & 25 & 31.1 & 46.7 & $38.0 \pm 4.4$ & 1.7 \\
\hline LFE-LTC & 20 & 27.2 & 49.0 & $38.7 \pm 6.5$ & 2.9 & 25 & 20.4 & 50.3 & $37.6 \pm 6.8$ & 2.6 \\
\hline
\end{tabular}

Measurements in mm. N, sample size; Min, minimum; Max, maximum; SD, standard deviation; CI, confidence interval; MFE, medial most point of the medial epicondyle of the femur; LFE, lateral most point of the lateral epicondyle of the femur; MTC, medial most point of the medial condyle of the tibia; LTC, lateral most point of the lateral condyle of the tibia.

Table 3. The measured distances of each neurovascular structure from the medial bony landmarks in male and female cadavers

\begin{tabular}{|c|c|c|c|c|c|c|c|c|c|c|c|}
\hline & \multirow{2}{*}{ Measurement } & \multicolumn{5}{|c|}{ Male } & \multicolumn{5}{|c|}{ Female } \\
\hline & & $\mathrm{N}$ & Min & Max & Mean \pm SD & $95 \% \mathrm{CI}$ & $\mathrm{N}$ & Min & $\operatorname{Max}$ & Mean \pm SD & $95 \% \mathrm{CI}$ \\
\hline \multirow[t]{3}{*}{ SSV } & M5 & 20 & 19.4 & 77.2 & $37.6 \pm 12.5$ & 5.5 & 25 & 16.1 & 50.6 & $37.9 \pm 8.2$ & 3.2 \\
\hline & M6 & 20 & 16.5 & 76.5 & $34.2 \pm 13.4$ & 5.9 & 25 & 14.3 & 54.0 & $34.2 \pm 9.0$ & 3.5 \\
\hline & M7 & 20 & 10.9 & 74.2 & $31.3 \pm 14.3$ & 6.3 & 25 & 11.2 & 56.3 & $30.1 \pm 10.5$ & 4.1 \\
\hline \multirow[t]{3}{*}{ MCSN } & M8 & 19 & 16.8 & 75.5 & $39.2 \pm 14.0$ & 6.3 & 24 & 13.6 & 56.0 & $38.8 \pm 10.1$ & 4.0 \\
\hline & M9 & 19 & 13.8 & 70.4 & $36.0 \pm 13.2$ & 5.9 & 24 & 3.8 & 51.8 & $34.3 \pm 11.6$ & 4.6 \\
\hline & M10 & 19 & 8.5 & 61.9 & $32.4 \pm 12.7$ & 5.7 & 24 & 19.7 & 51.7 & $33.1 \pm 10.0$ & 4.0 \\
\hline \multirow[t]{3}{*}{$\mathrm{TN}$} & M14 & 20 & 14.2 & 62.5 & $39.4 \pm 10.2$ & 4.5 & 25 & 23.9 & 54.7 & $38.0 \pm 8.1$ & 3.2 \\
\hline & M15 & 20 & 15.1 & 57.0 & $35.0 \pm 8.8$ & 3.8 & 25 & 22.6 & 47.4 & $34.8 \pm 7.3$ & 2.9 \\
\hline & M16 & 20 & 17.7 & 47.0 & $30.9 \pm 7.8$ & 3.4 & 25 & 17.4 & 42.1 & $31.4 \pm 6.3$ & 2.5 \\
\hline \multirow[t]{3}{*}{ PV } & M20 & 20 & 12.7 & 70.5 & $38.4 \pm 12.9$ & 5.6 & 25 & 22.6 & 42.1 & $32.8 \pm 5.6$ & 2.2 \\
\hline & M21 & 20 & 16.4 & 64.6 & $35.6 \pm 12.2$ & 5.4 & 25 & 16.8 & 44.3 & $31.3 \pm 6.4$ & 2.5 \\
\hline & M22 & 20 & 17.7 & 55.6 & $32.4 \pm 11.8$ & 5.2 & 25 & 13.0 & 45.3 & $30.2 \pm 7.5$ & 2.9 \\
\hline \multirow[t]{3}{*}{ PA } & M23 & 20 & 14.1 & 79.0 & $38.4 \pm 12.1$ & 5.3 & 25 & 24.3 & 42.0 & $34.6 \pm 4.9$ & 1.9 \\
\hline & M24 & 20 & 21.1 & 67.5 & $36.4 \pm 9.6$ & 4.2 & 25 & 18.4 & 41.4 & $33.0 \pm 6.1$ & 2.4 \\
\hline & M25 & 20 & 23.2 & 62.2 & $35.4 \pm 9.0$ & 3.9 & 25 & 19.1 & 43.8 & $32.8 \pm 7.1$ & 2.8 \\
\hline
\end{tabular}

Measurements in mm. N, sample size; Min, minimum; Max, maximum; SD, standard deviation; CI, confidence interval; SSV, small saphenous vein; MCSN, medial cutaneous sural nerve; TN, tibial nerve; PV, popliteal vein; PA, popliteal artery.

Table 4. The measured distances of each neurovascular structure from the lateral bony landmarks in male and female cadavers

\begin{tabular}{|c|c|c|c|c|c|c|c|c|c|c|c|}
\hline & \multirow{2}{*}{ Measurement } & \multicolumn{5}{|c|}{ Male } & \multicolumn{5}{|c|}{ Female } \\
\hline & & $\mathrm{N}$ & Min & $\operatorname{Max}$ & Mean \pm SD & $95 \% \mathrm{CI}$ & $\mathrm{N}$ & Min & Max & Mean \pm SD & $95 \% \mathrm{CI}$ \\
\hline \multirow[t]{3}{*}{ LCSN } & M11 & 12 & 15.1 & 43.1 & $24.9 \pm 7.3$ & 4.1 & 19 & 0.0 & 49.6 & $18.4 \pm 10.4$ & 4.7 \\
\hline & M12 & 12 & 15.0 & 47.7 & $25.9 \pm 9.0$ & 5.1 & 19 & 2.0 & 44.6 & $18.2 \pm 9.6$ & 4.3 \\
\hline & M13 & 12 & 16.7 & 50.9 & $27.3 \pm 10.8$ & 6.1 & 19 & 6.3 & 40.3 & $19.9 \pm 9.0$ & 4.1 \\
\hline \multirow[t]{3}{*}{$\mathrm{CFN}$} & M17 & 20 & 0.0 & 37.0 & $13.4 \pm 8.2$ & 3.6 & 25 & 0.0 & 46.4 & $8.4 \pm 9.1$ & 3.6 \\
\hline & M18 & 20 & 0.0 & 27.6 & $10.3 \pm 6.6$ & 2.9 & 25 & 0.0 & 38.8 & $6.7 \pm 7.8$ & 3.1 \\
\hline & M19 & 20 & 0.0 & 18.1 & $7.7 \pm 4.8$ & 2.1 & 25 & 0.0 & 26.0 & $5.3 \pm 6.0$ & 2.4 \\
\hline
\end{tabular}

Measurements in mm. N, sample size; Min, minimum; Max, maximum; SD, standard deviation; CI, confidence interval; LCSN, lateral cutaneous sural nerve; CFN, common fibular nerve. 
Table 5. The mean deviation and direction of deviation for each neurovascular structure within the popliteal fossa in males and females

\begin{tabular}{lccccc}
\hline \multirow{2}{*}{ Structure } & \multicolumn{3}{c}{ Male } & & \multicolumn{2}{c}{ Female } \\
\cline { 2 - 3 } \cline { 5 - 6 } & Deviation & Direction & & Deviation & Direction \\
\hline SSV & 6.3 & Medial & & 7.8 & Medial \\
MCSN & 6.8 & Medial & & 5.7 & Medial \\
LCSN & 2.4 & Medial & & 1.5 & Medial \\
TN & 8.5 & Medial & & 6.6 & Medial \\
CFN & 5.7 & Lateral & & 3.1 & Lateral \\
PV & 6.0 & Medial & & 2.6 & Medial \\
PA & 3.0 & Medial & & 1.8 & Medial \\
\hline
\end{tabular}

Measurements in mm. SSV, small saphenous vein; MCSN, medial cutaneous sural nerve; LCSN, lateral cutaneous sural nerve; TN, tibial nerve; CFN, common fibular nerve; PV, popliteal vein; PA, popliteal artery.

ral bony landmark proximally and the tibial bony landmark distally (Table 5). These results demonstrate the course of the neurovascular structure through the popliteal fossa and highlight the differences in neurovascular relations between the femoral and tibial plane. All structures deviated medially when passing through the popliteal fossa with exception to the CFN which deviated laterally (Table 5).

No correlation was found between weight and the femoral epicondylar distance $(\mathrm{r}=-0.004)$ or the tibial condylar distance $(r=-0.09)$. There was a moderate correlation between height and the femoral epicondylar distance $(r=0.6)$ and between height and tibial intercondylar distance $(r=0.5)$. The distance between the two femoral epicondyles or tibial condyles showed no or very little correlation to the distance of the neurovascular structures from those bony landmarks $(\mathrm{r}=0.10-0.38)$.

\section{Discussion}

This study defined the popliteal fossa by reliable bony landmarks and provided a comprehensive map of the neurovascular structures which can be applied in direct posterior surgical access to the knee. The 'inverted L-shape' incision used for posterolateral tibial plateau fractures is traced along the medial edge of the biceps femoris muscle spanning over the articulate line [5]. The positioning of the CFN relative to this landmark is pertinent in protecting the nerve. The mean distance between the LFE and CFN in this study was 10.6 $\mathrm{mm}, 10.3 \mathrm{~mm}$ at the midpoint between LFE and LCT and 6.4 $\mathrm{mm}$ at the LTC. In 9 of the 45 knees, the CFN was traversing directly over either the LFE or LTC or both. There is very limited research available on measurements of the CFN from the bony landmarks used in this study. Thi et al. [6] demon- strated a mean distance between the lateral femoral condyle and the CFN of $29.6 \mathrm{~mm}$, which is substantially more medial when compared to a mean of $11 \mathrm{~mm}$ in this study. Unfortunately, Thi et al. [6] have failed to specify the exact anatomic landmark they have utilized for measurement, making comparisons extremely difficult.

Median incisions must take the underlying popliteal neurovascular bundle layout into consideration. The popliteal neurovascular bundle consists of, superficial to deep, the TN, PV and PA [7]. The TN lies laterally in relation to the PA and PV [8]. The popliteal neurovascular bundle courses deep to and in between the two heads of the gastrocnemius muscle. The TN lies superficially in the bundle; therefore, surgeons must practice care during incision and dissection to avoid iatrogenic damage to the nerve. The distance between the MFE and the TN is $33-45 \mathrm{~mm}$ in males and $34-42 \mathrm{~mm}$ in females. Sora et al. [9] measured the distance between the MFE and the TN to be 51.1-52.2 mm. The PV and PA is lying deep to the TN and Sora et al. [9] reported the distances between the MFE and the medial borders of the PA and PV to be $42.3-49.7 \mathrm{~mm}$ and $46.0-53.5$ respectively. The findings of this study documented distances of 33-45 $\mathrm{mm}$ and $31-34$ $\mathrm{mm}$ respectively for males and $33-38 \mathrm{~mm}$ and $30-36 \mathrm{~mm}$ respectively for females. The ranges of mean distances between the MFE and the above-mentioned structures were lower than the ones defined by Sora et al. [9]. This explains the larger difference between Sora et al.'s results and this study's female results due to the significant difference between male and female knees. In addition, Sora et al. [9] measured frozen cadaveric knees that had been sliced and scanned whereas this study measured embalmed cadaveric knees.

The TN, PV and PA all deviated medially within the popliteal fossa. Between the femoral epicondylar level and the tibial condyle level the TN, PV and PA deviated medially by $8.5 \mathrm{~mm}, 6.0 \mathrm{~mm}$ and $3.0 \mathrm{~mm}$ respectively in males. In females the TN, PV and PA deviated medially by $6.6 \mathrm{~mm}$, $2.6 \mathrm{~mm}$ and $1.8 \mathrm{~mm}$ respectively. This implies that the more inferior aspect of the popliteal fossa will have a smaller medial surgical safe zone due to the course of these three structures. Similarly, Sora et al. [9] also noticed medial deviation of these structures. However, Sora et al. [9] utilized different landmarks making direct comparisons quite difficult.

The very similar male and female distances between medial bony landmarks and the SSV, MCSN and TN indicate that these neurovascular structures lie more medially in the male popliteal fossa than in the female. The difference in 
relative positioning between these neurovascular structures could be due to sexual dimorphism. The positioning of nerves in the popliteal fossa have been found to show significant differences between male and female after puberty due to the formation of muscle mass [10]. Along with the relative positioning differences displayed between male and female, the superficial structures; the SSV, LCSN and MCSN display a high level of variation. The LCSN and MCSN in particular are highly variable in pattern and presence [11, 12]. Incisions made in the median plane should therefore, factor in sex and sural nerve pattern variability.

The distances between bony landmark parameters in male and female knees were significantly larger by an average of $7 \%$ to $8 \%$ with the exception for the distance between LFE and LTC (3\%). This can possibly be explained by the difference in Q-angle between males and females. Females tend to have a larger Q-angle, therefore placing the LEF and the LTC closer together than observed in males [13].

Hafez et al. [14] compared mediolateral dimensions at the femoral condyles and tibial plateaus and reported smaller dimensions for both femoral epicondyle and tibial plateau distances in an Arabic population. Similarly, Miyatake et al. [15] measured mediolateral dimensions in Japanese patients and also reported smaller dimensions. Hafez et al. [14] compared their results to other population groups and were able to show that European groups had larger knees when compared to Asian groups highlighting possible population differences. However, both studies also demonstrated that the measures in females are consistently smaller compared to males and their ratios $(6 \%-9 \%)$ were comparable to the results of this study (6\%-8\%). Sexual dimorphism is the obvious reason for these findings as males are generally larger and more robust than females [16].

This study has several limitations. Due to the characteristically stiff cadaveric knees, superficial dissection is difficult, and it cannot be entirely excluded that MCSN, LCSN and SSV were compromised which would have resulted in different measures. However, on careful inspection of all 45 specimens, macroscopic damage to these structures was not observed. Photographs were taken at every level of the knee dissection to measure the structures on ImageJ. Whilst great care was taken to ensure reliable and reproducible images, it is possible that measurement bias was introduced, which may have influenced measures. Inclusion and exclusion criteria of this study were strictly defined, but it cannot entirely exclude the possibility that the neurovascular structures within the posterior knee had pathology that was not detected on macroscopic inspection. It is acknowledged that embalmment of human tissue results in significant stiffness when compared to fresh or fresh frozen tissue [17]. However, it is unlikely that these biomechanical changes influence tissue anatomy unless viscoelastic properties are investigated [17]. Theoretically embalmment could also result in changes of anatomic morphology and increasing difficulty with dissection [18]. However, Kennel et al. [19] were able to demonstrate that embalming was unlikely to influence dissection, tissue handling and the anatomy.

This study defined the popliteal fossa by reliable bony landmarks and provided a comprehensive map of the neurovascular structures. This will assist surgeons in avoiding injuries to important neurovascular structures during surgical procedures by using reliable and clearly identifiable landmarks and measure distances between these landmarks and the neurovascular structures in the popliteal fossa.

\section{ORCID}

Kelsi Greenwood: https://orcid.org/0000-0002-3923-7828

Reinette van Zyl: https://orcid.org/0000-0002-5059-1000

Natalie Keough: https://orcid.org/0000-0001-8249-8727

Erik Hohmann: https://orcid.org/0000-0003-1991-8682

\section{Author Contributions}

Conceptualization: KG, RvZ, EH. Data acquisition: KG. Data analysis or interpretation: KG, NK, EH. Drafting of the manuscript: KG, RvZ. Critical revision of the manuscript: NK, EH. Approval of the final version of the manuscript: all authors.

\section{Conflicts of Interest}

No potential conflict of interest relevant to this article was reported.

\section{References}

1. Faucett SC, Gannon J, Chahla J, Ferrari MB, LaPrade RF. Posterior surgical approach to the knee. Arthrosc Tech 2017;6:e3915.

2. Alpert JM, McCarty LP, Bach BR Jr. The direct posterior approach to the knee: surgical and anatomic approach. J Knee Surg 2008;21:44-9. 
3. Dagur G, Gandhi J, Smith N, Khan SA. Anatomical approach to clinical problems of popliteal fossa. Curr Rheumatol Rev 2017;13:126-38.

4. Landis JR, Koch GG. The measurement of observer agreement for categorical data. Biometrics 1977;33:159-74.

5. Chen H, Wu L. Surgical options for posterior tibial plateau fracture. Int J Clin Exp Med 2015;8:21421-7.

6. Thi C, Van Huy N, Nguyen NC, Thanh TH. Applied anatomy of common peroneal nerve: a cadaveric study. Int J Med Pharm 2018;6:6-10.

7. Hyland S, Sinkler MA, Varacallo M. Anatomy, bony pelvis and lower limb, popliteal region. Treasure Island: StatPearls Publishing; 2020.

8. Kim TK, Savino RM, McFarland EG, Cosgarea AJ. Neurovascular complications of knee arthroscopy. Am J Sports Med 2002;30:619-29.

9. Sora MC, Dresenkamp J, Gabriel A, Matusz P, Wengert GJ, Bartl R. The relationship of neurovascular structures to the posterior medial aspect of the knee: an anatomic study using plastinated cross-sections. Rom J Morphol Embryol 2015;56:1035-41.

10. Byun S, Gordon J, Morris S, Jacob T, Pather N. A computed tomography and magnetic resonance imaging study of the variations of the sciatic nerve branches of the pediatric knee: implications for peripheral nerve blockade. Clin Anat 2019;32:83650.

11. Aktan Ikiz ZA, Uçerler H, Bilge O. The anatomic features of the sural nerve with an emphasis on its clinical importance.
Foot Ankle Int 2005;26:560-7.

12. Paraskevas GK, Natsis K, Tzika M, Ioannidis O. Fascial entrapment of the sural nerve and its clinical relevance. Anat Cell Biol 2014;47:144-7.

13. Nguyen AD, Boling MC, Levine B, Shultz SJ. Relationships between lower extremity alignment and the quadriceps angle. Clin J Sport Med 2009;19:201-6.

14. Hafez MA, Sheikhedrees SM, Saweeres ES. Anthropometry of Arabian arthritic knees: comparison to other ethnic groups and implant dimensions. J Arthroplasty 2016;31:1109-16.

15. Miyatake N, Sugita T, Aizawa T, Sasaki A, Maeda I, Kamimura M, Fujisawa H, Takahashi A. Comparison of intraoperative anthropometric measurements of the proximal tibia and tibial component in total knee arthroplasty. J Orthop Sci 2016;21:6359.

16. Ubelaker DH, DeGaglia CM. Population variation in skeletal sexual dimorphism. Forensic Sci Int 2017;278:407.e1-7.

17. Hohmann E, Keough N, Glatt V, Tetsworth K, Putz R, Imhoff A. The mechanical properties of fresh versus fresh/frozen and preserved (thiel and formalin) long head of biceps tendons: a cadaveric investigation. Ann Anat 2019;221:186-91.

18. Eisma R, Lamb C, Soames RW. From formalin to thiel embalming: what changes? One anatomy department's experiences. Clin Anat 2013;26:564-71.

19. Kennel L, Martin DMA, Shaw H, Wilkinson T. Learning anatomy through thiel- vs. formalin-embalmed cadavers: student perceptions of embalming methods and effect on functional anatomy knowledge. Anat Sci Educ 2018;11:166-74. 